\title{
Upregulation of microRNA-200a associates with tumor proliferation, CSCs phenotype and chemosensitivity in ovarian cancer
}

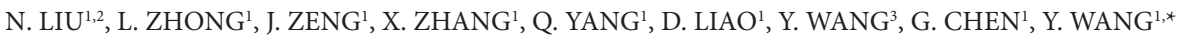 \\ ${ }^{1}$ Department of Obstetrics and Gynecology, Zhujiang Hospital, Southern Medical University, Guangzhou, China; ${ }^{2}$ Department of Gynecology, \\ Shanghai First Maternity and Infant Hospital Affiliated to Tong Ji University, Shanghai, China; ${ }^{3}$ School of Basic Medical Sciences, Cancer \\ Research Institute, Southern Medical University, Guangzhou, China
}

*Correspondence: yifengw1963@sina.com

Received October 3, 2014 / Accepted January 4, 2015

\begin{abstract}
Ovarian cancer is a lethal gynecologic malignancy and always has a poor prognosis. Despite new treatments modalities, the long term outcomes had not been significantly improved in the past 30 years. Although microRNA-200a (miR-200a) has been reported to be a prognostic marker in ovarian cancer, it's exact role in ovarian cancer remain unclear. In this study, we inserted the response element of miR-200a in ovarian cancer cell line via lentivirus-mediated transgene in vitro, and qRT-PCR (real time quantitative reverse transcription PCR) assay confirmed that miR-200a was up regulated compared with control. Then colony-formation assay, cell cycle analysis, CCK8 assays in vitro and xenograft experiments in vivo were performed and verified that miR-200a promoted proliferation, while blocked the formation of tumor spheroids and reduced the ratio of SP (side population) cells in ovarian cancer. Finally, we validated that miR-200a significantly enhanced the chemosensitivity of paclitaxel but not cisplatin in both adherent culture and sphere culture. Taken together, we demonstrated that upregulation miR-200a promoted proliferation and inhibited cancer stem cells (CSCs) phenotype in OVCAR-3 ovarian cancer cell line, combined with cell cycle-targeting drug paclitaxel could effectively eliminate the "side effects" of proliferation, and showed evidences that this strategy may be promising for ovarian cancer treatment.
\end{abstract}

Key words: microRNA, ovarian cancer, proliferation, cancer stem cells, chemosensitivity

Ovarian cancer is still the most lethal gynaecologic malignancy according to 2013 statistics [1]. Although the current standard treatment, systemic by administration of platinum and paclitaxel after debulking surgery was efficient in almost $70 \%$ of ovarian cancer cases, most of these patients ultimately develop chemoresistance and recurrence [2].

Recently, accumulated evidence showed that the miR-200a expression level was closely associated with progression free survival (PFS), overall survival (OS) and chemo-sensitivity in ovarian cancer, thus miR-200a was suitable as a prognostic marker for ovarian cancer outcome $[3,4]$. Previously, we demonstrated that the profile of miRNAs was different between $\mathrm{CD} 133^{+}$and $\mathrm{CD} 133^{-}$ovarian cancer cells, miR-200a was down-regulated in $\mathrm{CD}_{133^{+}}$ovarian cancer stem cells (CSCs) [5], and upregulation miR-200a could reduce ZEB2-mediated migration and invasion of $\mathrm{CD}_{133^{+}}$ovarian CSCs [6]. However, miR-200a regulation of proliferation, CSCs phenotype and chemosensitivity in ovarian cancer remain unclear. Therefore, an in-depth understanding of the roles and mechanisms of miR-200a in ovarian cancer is needed.

Herein, we first established that miR-200a was stably overexpressed in OVCAR-3 ovarian cancer cell line and then validated that over-expression of miR-200a increased tumor proliferation and tumor volume. Additionally, we found that the over-expression of miR-200a weakened the CSCs phenotype by blocking the formation of tumor spheroids and reducing the ratio of side population (SP) cells. Importantly, we demonstrated miR-200a upregulation combined with cell cycle-targeting drug paclitaxel could effectively eliminate the "side effects" of proliferation in both adherent culture and sphere culture, and this strategy may be promising for ovarian cancer treatment.

\section{Materials and methods}

Adherent and suspension cells culture. The human ovarian cancer cell line OVCAR-3 (NIH) was cultured in 
RPMI-1640 (Hyclone) supplemented with 10\% foetal bovine serum (FBS; PAA), $100 \mathrm{U} / \mathrm{mL}$ penicillin and $100 \mu \mathrm{g} /$ $\mathrm{mL}$ streptomycin (PAA). The spheroids were cultured as follows: cells were plated in ultra-low attachment petri dishes (Corning) at a density of $5 \times 10^{4}$ cells $/ \mathrm{mL}$ in stem cell medium (SCM) [DMEM/F12 (1:1, GIBICO) supplemented with $0.4 \%$ bovine serum albumin (BSA; Sigma), $20 \mathrm{ng} / \mathrm{mL}$ epidermal growth factor (EGF; PeproTech), $10 \mathrm{ng} / \mathrm{mL}$ basic fibroblast growth factor (bFGF; PeproTech), $5 \mu \mathrm{g} / \mathrm{mL}$ insulin (Sigma), and $100 \mathrm{U} / \mathrm{mL}$ penicillin and $100 \mu \mathrm{g} / \mathrm{mL}$ streptomycin (PAA)]. Spheroids were dissociated every week using $0.25 \%$ trypsin/0.02\% EDTA solution (Sigma) for $2 \mathrm{~min}$ at room temperature. Cells were incubated at $37^{\circ} \mathrm{C}$ in a humidified chamber supplemented with $5 \% \mathrm{CO}_{2}$.

Establishment of miR-200a overexpressing ovarian cancer cells. Lentiviral expression clones for miR-200a and control miRNA (pLV-hsa-miR-200a and pLV-miR-control) were purchased from HAPK (ShenZhen, China), while the lentiviral packaging plasmids (psPAX2 and pMD2.G) were kindly provided by Dr. Didier Trono (University of Geneva, Switzerland). To produce viruses expressing empty vector or miR-200a, pLV-miR-control or pLV-hsa-miR-200a was cotransfected with the packaging plasmids psPAX2 and pMD2.G into HEK293T cells using Lipofectamine 2000 (Invitrogen) according to protocol. The packaged lentiviruses expressing empty vector and miR-200a were named LV-Con and LV-miR-200a, respectively (Supplementary Figure S1). The supernatant was harvested $60-72 \mathrm{~h}$ after transfection, and then used to infect OVCAR-3 cells with $10 \mu \mathrm{g} / \mathrm{mL}$ polybrene (Sigma). GFP (green fluorescent protein) expressing cells were sorted by FACS Aria II (BD, USA). The levels of miR200a were verified using qRT-PCR.

RNA extraction and qRT-PCR. Total RNA was extracted using Trizol Reagent (Invitrogen) according to protocol, and reverse-transcribed into cDNA using the One Step PrimeScript miRNA cDNA Synthesis Kit (TaKaRa). qRT-PCR was carried out using SYBR Green qRT-PCR master mix (TaKaRa). U6 level was used as internal control. The comparative $\mathrm{Ct}(\Delta \Delta \mathrm{Ct})$ method was use to determine the expression fold change. The following primers used for qRT-PCR were miR-200a: TAACACTGTCTGGTAAC GATGT, U6: CTCGCTTCGGCAGCACA.

Colony formation assay. OVCAR-3/Con and OVCAR-3/ miR-200a cells in 2D culture were plated in 6-well culture plates at 150 cells/well. After incubation for $10 \mathrm{~d}$, cells were washed twice with PBS and stained with haematoxylin solution. The number of colonies containing $\geq 50$ cells was counted under a microscope. The colony-formation efficiency was calculated as: (number of colonies/number of cells inoculated) $\times 100 \%$.

Cell cycle analysis. OVCAR-3/Con and OVCAR-3/miR200a cells in adherent culture and sphere culture were rinsed with cold PBS (phosphate-buffered saline), respectively. Then fixed with $70 \%$ ice-cold ethanol for $24 \mathrm{~h}$ at $4{ }^{\circ} \mathrm{C}$, and rinsed with cold PBS, followed by incubation with PBS containing
$50 \mu \mathrm{g} / \mathrm{mL}$ propidium iodide (PI) and $100 \mu \mathrm{g} / \mathrm{mL}$ of RNase A for 30 min in the dark. Finally, the DNA content of the labelled cells was acquired using FACS Aria II (BD, USA).

CCK8 assay. OVCAR-3/Con or OVCAR-3/miR-200a cells in adherent culture and sphere culture were seeded at 1,000 single cells per well into 96 -well plates containing $100 \mu \mathrm{L}$ of RPMI- 1640 supplemented with 10\% FBS, and 4,000 single cells per well into 96-well ultra low plates (Corning) containing 100 $\mu \mathrm{L}$ of SCM, respectively. The viable cell numbers were measured at fixed times according to the protocol of Cell Counting Kit-8 (Dojin Laboratories, Japan); the absorbance value (OD) of each well was measured at $450 \mathrm{~nm}$ (excitation).

In vivo xenograft experiments. Nude mice experiments were performed in accordance with the Institutional Animal Care and Use Committee procedures and guidelines of Zhujiang Hospital, Southern Medical University. $1.5 \times 10^{6}$ OVCAR-3/Con and OVCAR-3/miR-200a cells were injected subcutaneously in the back of 5-6 weeks BALB/c-nu/nu female mice, at left and right side, respectively, $\mathrm{n}=8$. Tumor volume was monitored every $3 \mathrm{~d}$ up to $62 \mathrm{~d}$ and calculated using the equation $\mathrm{V}\left(\mathrm{mm}^{3}\right)=\mathrm{a} \times \mathrm{b}^{2} / 2$, where $\mathrm{a}$ is the largest diameter, and $b$ is the perpendicular diameter.

Immunohistochemistry. Paraffin sections $(4 \mu \mathrm{m})$ from xenograft samples were deparaffinised according to a standard protocol. Heat-induced antigen retrieval was performed in $10 \mathrm{mM}$ citrate buffer for $2 \mathrm{~min}$ at $100^{\circ} \mathrm{C}$. Endogenous peroxidase activity and nonspecific antigens were blocked with peroxidase blocking reagent containing $3 \%$ hydrogen peroxide and serum, followed by incubation with rabbit anti-human Ki67 antibody (1:100, EPITOMICS, USA) overnight at $4^{\circ} \mathrm{C}$. After washing, sections were incubated with biotin-labelled goat anti-rabbit IgG antibody for $10 \mathrm{~min}$ at room temperature and were subsequently conjugated with HRP (Maixin, Inc., China). The peroxidase reaction was developed with 3,3-diaminobenzidine chromogen solution in 3,3'-diaminobenzidine (DAB) substrate buffer. Sections were visualised with DAB and counterstained with haematoxylin, mounted in neutral gum and analysed using a bright-field microscope. The stained tissue sections were reviewed and scored independently by two pathologists blinded to the clinical parameters. Staining localised to the cell nuclei was considered Ki67 positive. The number of positive-staining cells showing immunoreactivity in 10 representative microscopic fields was counted, and the percentage of positive cells was calculated.

Spheroid formation assay. OVCAR-3/Con and OVCAR-3/ miR-200a cells were seeded into 24-well ultra low plates (Corning) at 1,000 single cells per well in $1 \mathrm{~mL}$ of SCM for $7 \mathrm{~d}$. The number of tumor spheres with diameter $\geq 70 \mu \mathrm{m}$ was determined using an inverted fluorescence microscope (Nikon).

Side population assay. OVCAR-3/Con and OVCAR-3/ miR-200a cells were resuspended in RPMI- 1640 pre-warmed at $37^{\circ} \mathrm{C}$ and supplemented with $2 \%$ FBS. Hoechst 33342 $(5.0 \mathrm{mg} / \mathrm{mL}$; Sigma) in the presence or absence of verapamil (50 $\mu \mathrm{M}$; Sigma-Aldrich) as an inhibitor of the ATP-binding cassette blocks dye (Hoechs 33342) efflux. The cells were in- 
cubated at $37^{\circ} \mathrm{C}$ for 90 min with continuous shaking without exposure to light. PI $(1 \mu \mathrm{g} / \mathrm{mL})$ permits to exclude dead cells prior to the flow cytometric analysis by FACS Aria II (BD, USA). The Hoechst 33342 dye was excited at $357 \mathrm{~nm}$ and its fluorescence was analyzed using dual wave-lengths (blue, 402$446 \mathrm{~nm}$; red, 650-670 nm).

Drug chemosensitivity assay in adherent culture and sphere culture. Spheroids were dissociated using $0.25 \%$ trypsin/0.02\% EDTA solution and filtered through a $40 \mu \mathrm{m}$ cell strainer (BD). SDCs (spheroid-derived cells) were seeded into 96-well ultra low plates (Corning) at $1.0 \times 10^{4}$ /well in SCM. In parallel, OVCAR-3 cells were seeded into normal 96-well plates (Corning) at $5.0 \times 10^{3} /$ well in RPMI1640 medium containing 10\% FBS. After $24 \mathrm{~h}$, different concentrations of cisplatin and paclitaxel (Sigma) were added to different wells and cells were treated for a subsequent $72 \mathrm{~h}$. Thereafter, $20 \mu \mathrm{L}$ of MTT ( $5 \mathrm{mg} / \mathrm{mL}$; Sigma) was added to each well and incubated for $4 \mathrm{~h}$; at the end of incubation, 96-well ultra low plates were centrifuged in a box-type centrifuge at $3000 \mathrm{rpm}$ for $15 \mathrm{~min}$. Then, the supernatants of the SDCs and OVCAR-3 cells were removed carefully and $100 \mu \mathrm{L}$ of DMSO (Sigma) was added to each well. The absorbance value (OD) of each well was measured at $490 \mathrm{~nm}$ (excitation). The cells' viability was expressed as a percentage of the treated wells related to the untreated control wells $\times 100 \%$.

Statistical analysis. Statistical analysis was performed using a SPSS 16.0 software package. One-sample t-test was utilized to analyze the differential expression of miR-200a in adherent and suspension growth of ovarian cancer cells. Factorial design analysis of variance and two independent samples t-test was utilized to analyze the differences of cell viability in cytotoxicity tests, growth curves (CCK8) and xenograft in nude mice. In two independent samples t-test was utilized to analyze the differences of colony formation rate, Ki67-positive rate and cell cycle distribution. $P<0.05$ was considered statistically significant, all data are presented as "mean values \pm SD".

\section{Results}

Establishment of miR-200a over-expression in ovarian cancer cells. To obtain a miR-200a dysregulation model in ovarian cancer cells, both human miR-200a and GFP gene were introduced into OVCAR-3 ovarian cancer cell line via a lentiviral expression vector. The sequence information of plasmid pLV-hsa-miR-200a and pLV-miR-control are in the supplementary materials (sup. 1). After sorting by FACS, a nearly $95 \%$ infection efficiency was determined by GFP assay in both OVCAR-3/Con and OVCAR-3/miR-200a cells. The level of miR-200a was significantly higher in OVCAR-3/ miR-200a cells compared with control in adherent culture validated by qRT-PCR (Fig. 1A). Then, OVCAR-3/Con and OVCAR-3/miR-200a cells were plated into ultra-low attachment petri dishes in SCM to form tumor spheroids composed of multi-cells in a suspended mode. The level of miR-200a was also significantly higher in the SDCs/miR-200a compared with control in sphere culture validated by qRT-PCR (Fig. 1B). These results suggested that the stable ovarian cancer cell lines over-expressing miR-200a were successfully achieved in both adherent culture and sphere culture. Besides, we confirmed that the basal level of miR-200a in adherent growth of ovarian cancer cells was significantly higher than suspended growth cells (Supplementary Figure S2).

Overexpression of miR-200a facilitated the proliferation of ovarian cancer cells in vitro and in vivo. Colony formation assays, cell cycle analysis, CCK8 assays and xenograft experiments were utilised to verify the roles of miR-200a in proliferation. Ovarian cancer cells arranged in a more compact manner after accumulation of miR-200a, and the cloning efficiency was significantly increased in cells following restoration of miR-200a expression compared with control $(93.55 \pm 1.68 \%$ versus $79.78 \pm 3.08 \%, p<0.01$, Fig. $2 A)$. In addition, our results demonstrated that over-expression of miR-200a facilitated the G1 to $S$ and $G 2 / M$ transition in both adherent culture and sphere culture (Fig. 2B). Furthermore, the growth curves determined by the CCK8 assay showed that up-regulation of miR-200a significantly promoted proliferation in both adherent culture and sphere culture (Fig. 2C). These results suggested that the promoting effect of miR-200a facilitates both anchorage-dependent growth and cells growth without substrate attachment. Most importantly, over-expression of miR-200a significantly increased the tumor size in xenografted nude mice (Fig. 2D), and Ki-67 positive expression rate in subcutaneous xenograft tissue (Fig. 2E). Combining these results, we confirmed that miR-200a promotes the proliferation of ovarian cancer cells.

Overexpression of miR-200a weakened ovarian CSCs phenotype. In spheroid formation assay, many miR-200a over-expressed cells presented in "single" suspended state rather than control cells that aggregated to form tumor spheroids, and as shown in the histogram, restoration of miR-200a significantly reduced the tumor spheroid formation per 1000 cells compared with the control ( $17.67 \pm 1.53$ vs. $6.33 \pm 1.16, p<$ 0.01 , Fig. $3 \mathrm{~A}$ ). However, the size of the tumor spheroids was similar compared with the control. In addition, as presented in Fig. 3B, over-expression of miR-200a induced the attachment of SDCs, even in ultralow attachment dishes. Furthermore, we demonstrated that the proportion of SP cells was significantly reduced after the overexpression of miR-200a (Fig. 3C). These observations indicated that restoration of miR-200a is not conducive to maintain the CSCs phenotype in ovarian cancer.

Overexpression of miR-200a significantly enhanced the chemosensitivity of paclitaxel but not of cisplatin. To investigate whether restoration of miR-200a would affect the chemosensitivity of ovarian cancer, OVCAR-3/miR-200a and SDCs/miR-200a cells were exposed to cisplatin or paclitaxel in adherent culture and sphere culture, respectively. Over-expression of miR-200a significantly increased the chemosensitivity of paclitaxel but not cisplatin in both adherent culture and sphere culture compared with the control (Figs. 4A-B). The vi- 


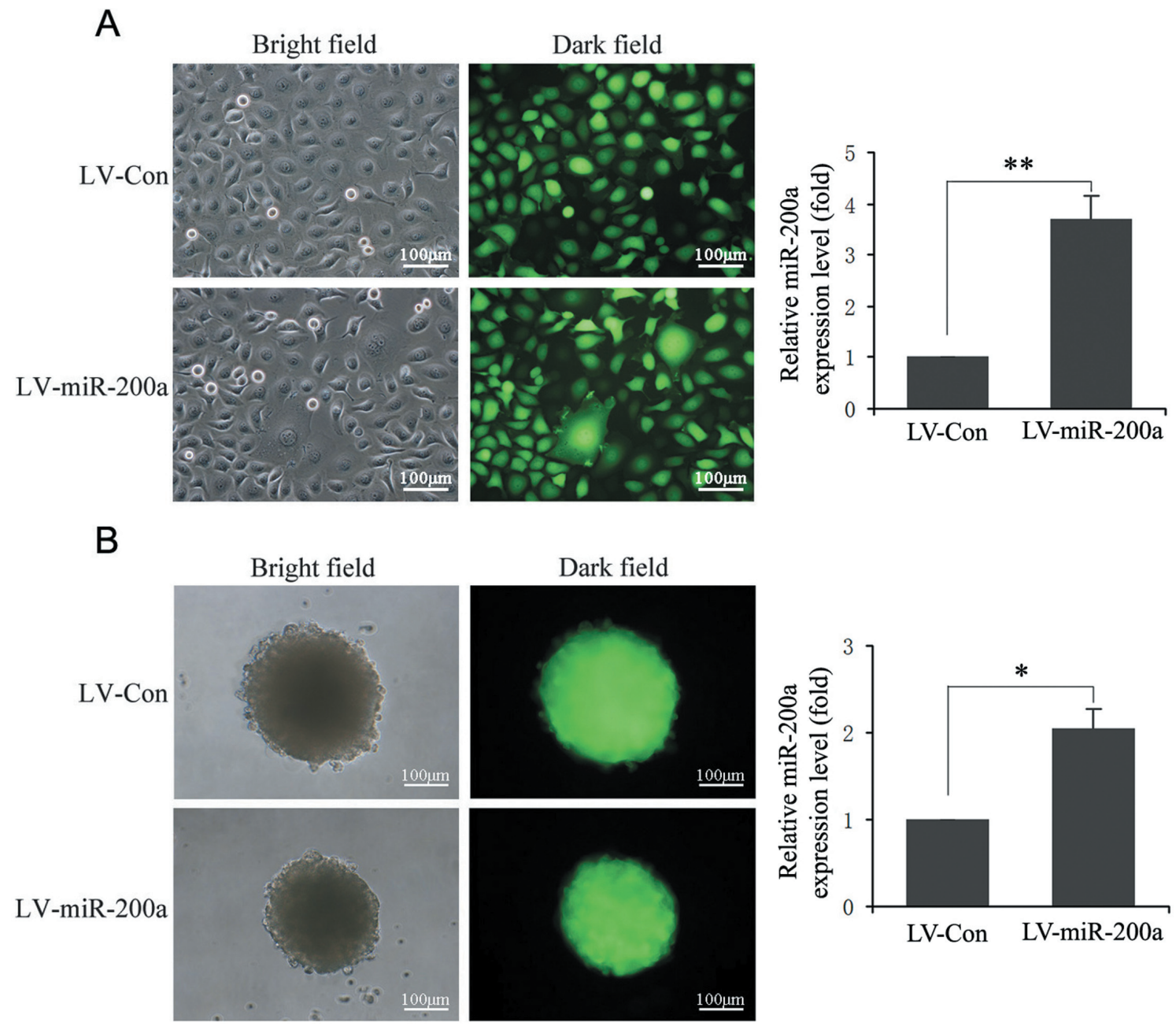

Figure 1. Over-expression of miR-200a in ovarian cancer cells. (A) Generation of stable OVCAR-3 cells expressing GFP and miR-200a transgenes in 2D culture. Left: GFP assay under inverted fluorescence microscopy; Right: qRT-PCR of the miR-200a. (B) Generation of stable SDCs expressing GFP and miR-200a transgenes in 3D culture. Left: GFP assay under inverted fluorescence microscopy; Right: qRT-PCR of the miR-200a.

abilities of the OVCAR-3/miR-200a and SDCs/miR-200a cells compared with the control were $(65.86 \pm 4.24 \%$ versus 75.15 $\pm 4.46 \%, p<0.01)$ and $(57.03 \pm 3.63 \%$ versus $78.91 \pm 4.01 \%$, $p<0.001$ ), respectively, when the concentration of paclitaxel was $8 \mathrm{nM}$. Interestingly, the chemosensitivity of paclitaxel was not significantly increased with the concentration of paclitaxel. It is well known that paclitaxel is a cell cycle dependent drug that induces cell apoptosis by over-stabilising microtubules, resulting in cell arrest at G2/M phase [7].

\section{Discussion}

Since 2006, an increasing number of studies have indicated an essential role of microRNAs in ovarian cancer. In addition to these specific regulators, miR-200 family (consisting of five members miR-200a/b/c, miR-141, miR-429) which is significantly involved in inhibition of epithelial-to-mesenchymal transition (EMT), repression of cancer stem cells (CSCs) selfrenewal and differentiation, modulation of cell division and apoptosis, and reversal of chemoresistance, is becoming a new star miRNA in study of human cancer [8].

In this study, we found that upregulation of miR-200a significantly promoted ovarian cancer cells proliferation in vitro and in vivo. This finding was consistent with Mateescu's group [9]. Then we'll ask the question: what is the relationship between proliferation and prognosis in ovarian cancer? Gene expression profiling data from 322 high-grade ovarian cancer cases found that proliferation-associated signalling pathways (MAPK signalling pathway, cell cycle, and cell proliferation pathways) were closely related to platinum/paclitaxel-based treatments [10]. Another study about The Cancer Genome Atlas (TCGA) project analysed 489 high-grade serous ovarian adenocarcinomas in 2011 found that patients with proliferative state survived significantly longer than patients with mesen- 


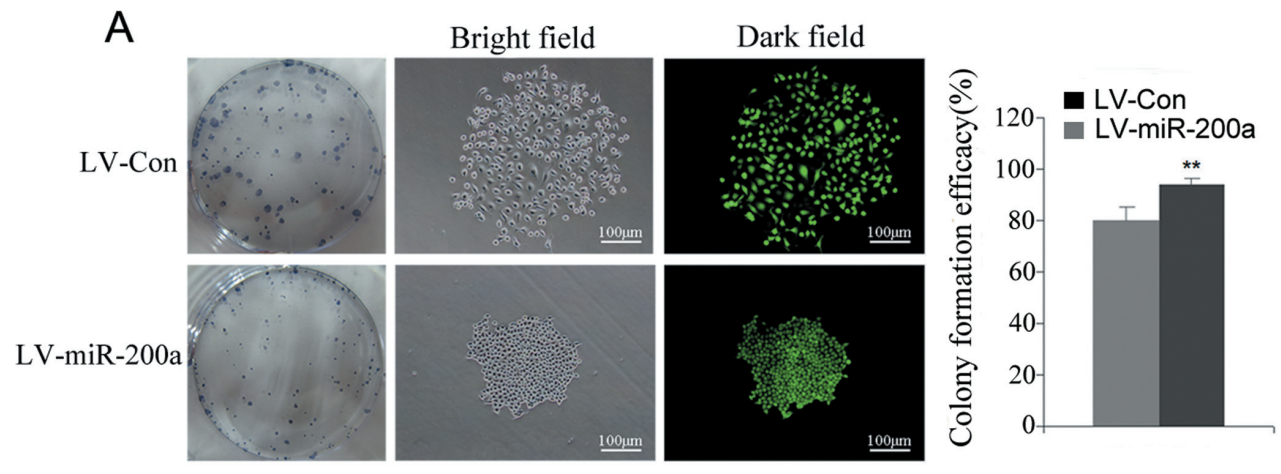

B
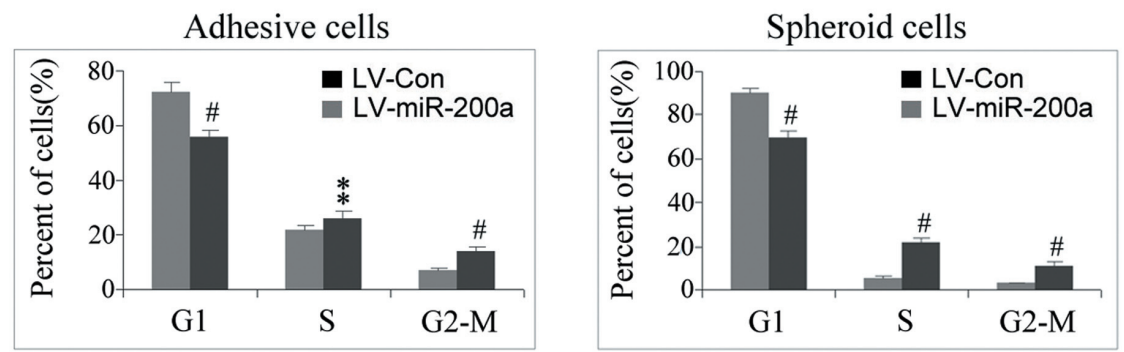

C

Adhesive cells
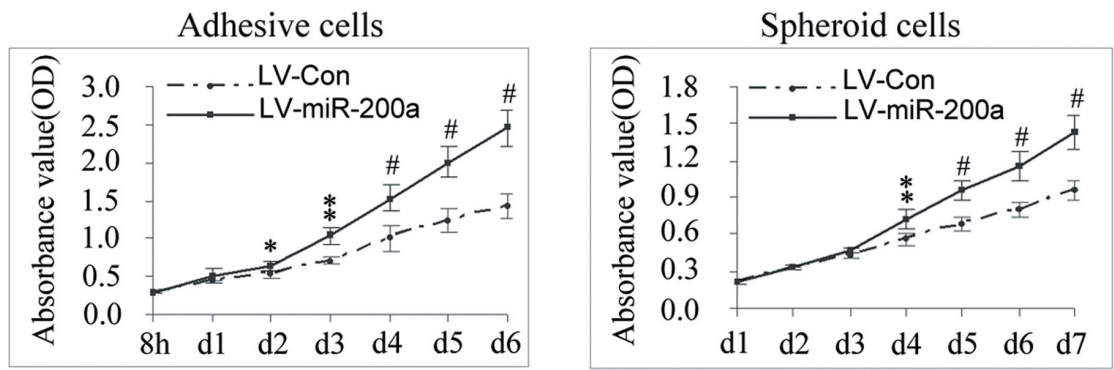

D
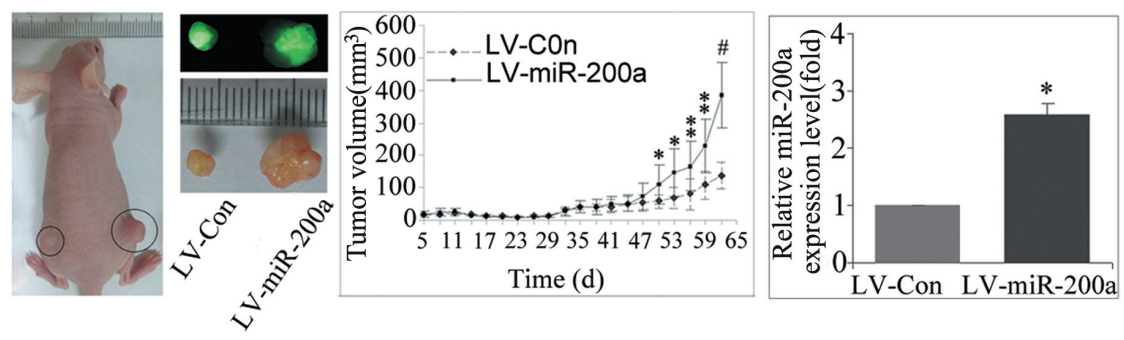

$\mathrm{E}$
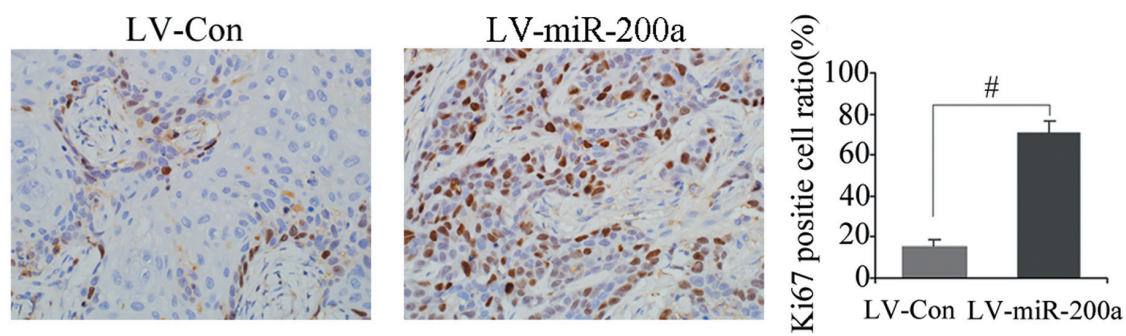

Figure 2. Over-expression of miR-200a promoted ovarian cancer cells proliferation in vitro and in vivo. (A) Colony formation assay in adhesive ovarian cancer cells. Left: morphology of cells clones. Right: the percentage of visible colonies. (B) Cell cycle distribution in adhesive and suspended ovarian cancer cells. (C) The growth curves of adhesive and suspended ovarian cancer cells by CCK8 assay. (D) In vivo xenograft experiments. Left: appearance of xenograft in nude mice. Middle: xenograft growth curves, $n=8$. Right: $q R T-P C R$ of miR-200a in xenografted tissue. (E) Immunohistochemical detection of Ki-67 expression in xenografted tissues. Original magnification, X 200. Left: the morphology of Ki-67 positive cells. Right: the percentage of Ki-67 positive cells. 
chymal subtype [11]. These findings indicated that the state of proliferation was not always a positive factor for the poor prognosis of ovarian cancer, due to it beneficial in increasing the sensitivity of chemotherapy.

Considering the central role of CSCs in tumorigenesis, we further explored the roles of miR-200a in regulation of ovarian CSCs. It's well known, analysis the capacity to form multi-cellular spheroids is a commonly used technique to evaluate the self-renewal potential of CSCs in many solid tumors [12]. In our present study, we found that the ability to form spheroids in sphere culture was markedly restricted after restoration of miR-200a expression. Additionally, the

A
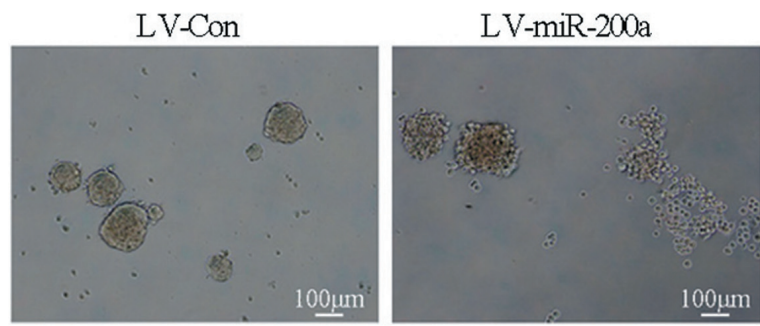

B

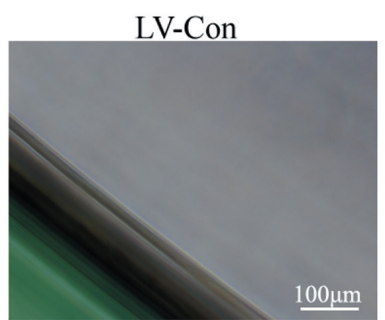

C
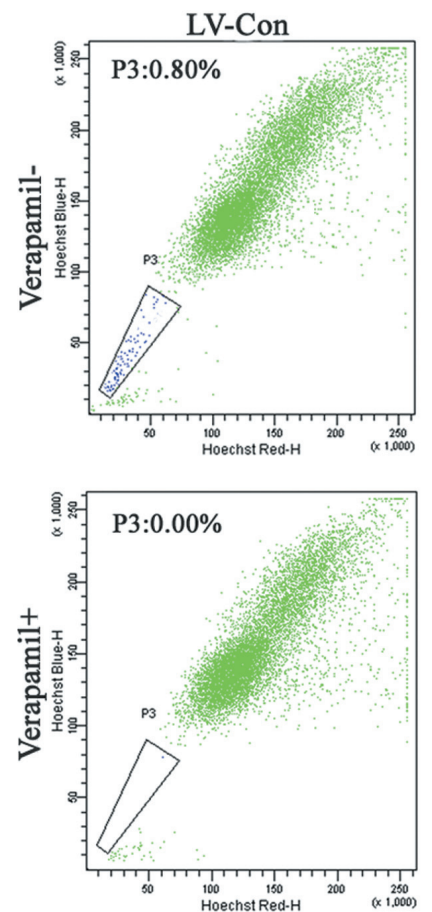

LV-miR-200a

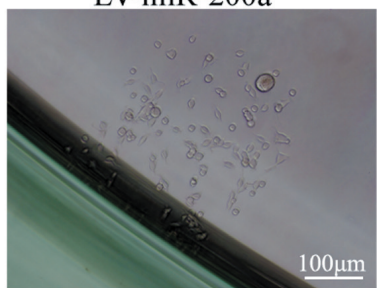

LV-miR-200a
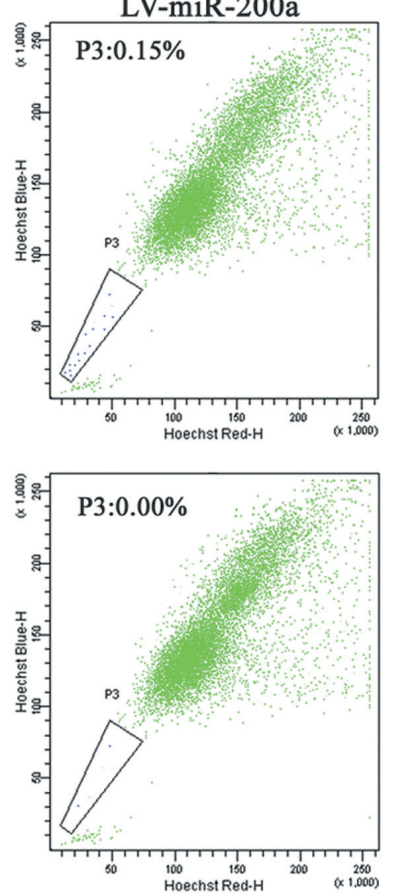

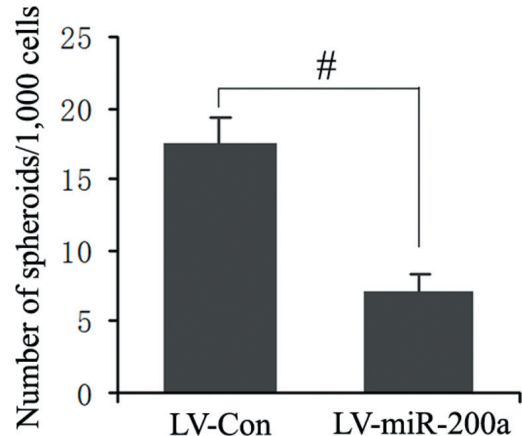

LV-Con LV-miR-200a

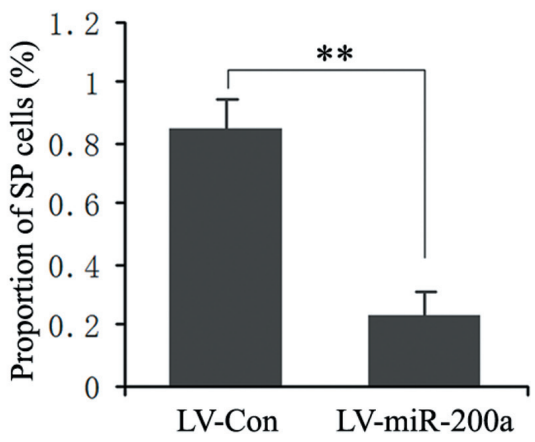

Figure 3. Over-expression of miR-200a hindered ovarian CSCs phenotype. (A) Spheroid formation assay. Left: images of tumor spheroids formation from 1,000 single ovarian cancer cells. Right: the number of tumor spheroids formed for each group. (B) The morphology of adherent cells in 3D culture. (C) Detection of SP cells from ovarian cancer cells. Left: SP cells were analyzed by FACS. Right: the ratio of SP cells. 
A
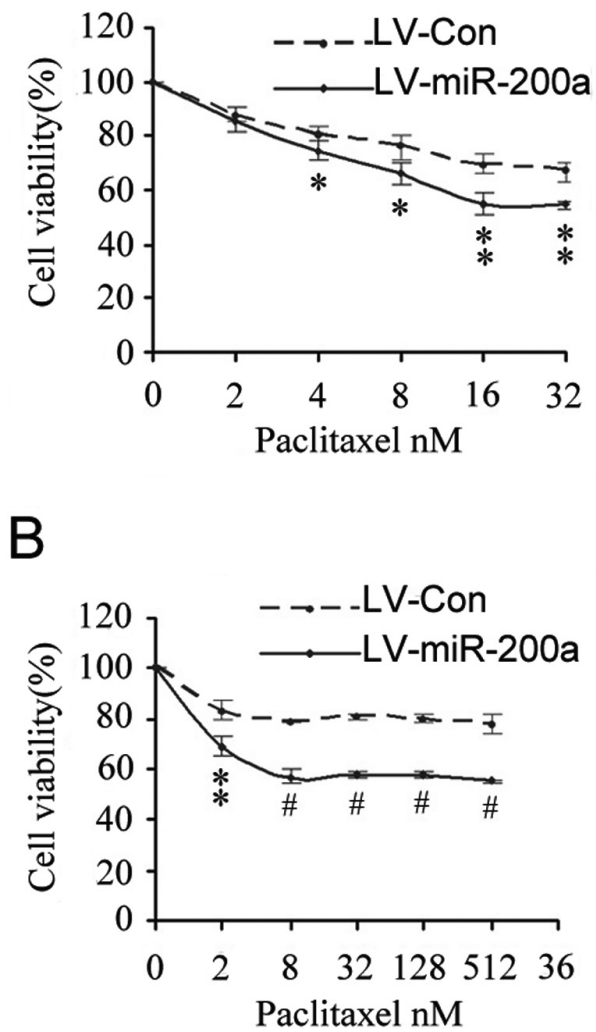
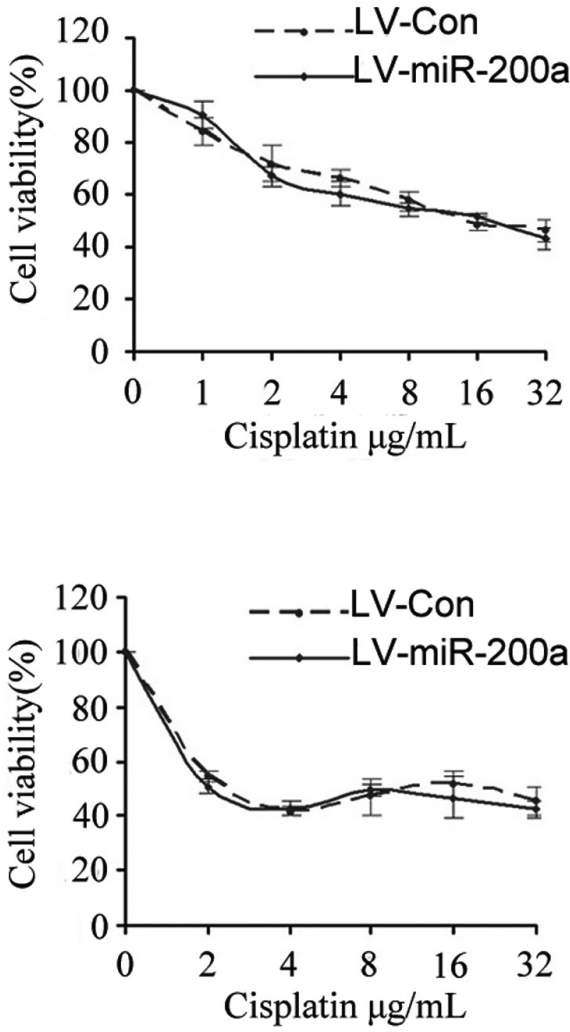

Figure 4. Over-expression of miR-200a enhanced the chemotoxicity of paclitaxel but not cisplatin in $2 \mathrm{D}$ and $3 \mathrm{D}$ culture. (A) Drug cytotoxicity assay in $2 \mathrm{D}$ culture. (B) Drug cytotoxicity assay in $3 \mathrm{D}$ culture.

tendency of suspended SDCs re-adhere to the plate surface was observed following the over-expression of miR-200a. Generally, SDCs enriched with poorly differentiated CSCs would remain suspended in serum-free medium [13]. Furthermore, overexpression of miR-200a significantly reduced the proportion of SP cells. Recently, some approaches have been described that SP cells exhibited higher tumorigenic ability and chemoresistance, thus suitable as a marker for ovarian CSCs $[14,15]$. Besides, we confirmed that the basal level of miR-200a in adherent growth of ovarian cancer cells was significantly higher than suspended growth cells. Considering our previous study validated that the expression level of miR-200a in $\mathrm{CD} 133^{+}$cells was lower than CD133- cells and the upregulated miR-200a could reduce ZEB2-mediated migration and invasion of $\mathrm{CD}_{13}{ }^{+} \mathrm{CSCs}$ [6]. These results indicated that the expression level of miR200a in CSCs is lower than non-CSCs, and over-expression of miR-200a was not conducive to maintaining the ovarian CSCs phenotype.

Some studies have confirmed that restoration of the miR200 family could effectively reverse chemoresistance to certain drugs; for example, restoration of miR-200c could enhance the sensitivity of paclitaxel by targeting TUBB3 [16], and overexpression of miR-200b could up-regulation of E2F3, which in turn contribute to the chemosensitivity of lung adenocarcinoma cells to docetaxel [17]. Since miR-200a has the role of promoting the proliferation of ovarian cancer, and there is a close relationship between proliferation and chemosensitivity, we further explored the effects of miR-200a to regulating the sensitivity of paclitaxel and cisplatin.

It was known that CSCs remained quiescent under steady state and that only a small fraction entered into the cell cycle, and this dormancy state is highly relevant to the efficacy of chemo-therapeutics, especially for cell cycle targeting drugs $[18,19]$. Wang's group found that SDCs in sphere culture maintained CSCs characteristics could be an ideal model for CSCs-targeted investigations in ovarian cancer [20]. Our research using this model found that SDCs in sphere culture were indeed a relatively quiet population and consistently showed high resistance to paclitaxel even at extremely large doses $(512 \mathrm{nM})$, and validated that after intensive expression of miR-200a, SDCs cell cycle progression were significantly accelerated and the chemosensitivity of paclitaxel was simultaneously and remarkably enhanced in sphere culture, but we did 
not found similar results in experiments with cisplatin. Studies have demonstrated that paclitaxel is an M phase-specific drug and may lose anticancer activity if the cancer cells were in the relative resting G0/G1 phase [21]. Our findings showed that inducing quiescent SDCs to enter into the cell cycle by upregulation of miR-200a might be an effective strategy for eliminating ovarian CSCs.

Besides, we observed that the toxic effects of paclitaxel in ovarian cancer cells seem to have certain limits. When cells' viability decreased to a certain extent, further increasing the concentration of paclitaxel did not effectively reduce the viability of the cells any more in adherent and sphere culture. This result was consistent with the clinical trial of Japanese GOG, in which they found dose-dense weekly paclitaxel (80 $\mathrm{mg} / \mathrm{m}^{2}$ ) with carboplatin treatment every 3 weeks improved both PFS (28 months vs. 17 months) and 3-year OS (72\% vs. $65 \%)$ when compared to paclitaxel $\left(180 \mathrm{mg} / \mathrm{m}^{2}\right)$ with carboplatin given every 3 weeks [22].

In conclusion, we demonstrated that restoration of miR-200a promoted proliferation while hindered the CSCs phenotype and enhanced the chemotoxicity of paclitaxel in ovarian cancer. Therefore, combination of overexpression miR-200a with cell cycle-targeting drugs to eliminating the effects of proliferation may generate a more positive outcome for ovarian cancer.

Supplementary information is available in the online version of the paper.

Acknowledgements: The authors thank Ying Wang for her helpful advices with the experiment and careful reading of this paper. This work was supported by the Fund for Technological Innovation Projects in the Department of Education of Guangdong, China (No. 2013KJCX0042) and the Talent Introduction Fund of Zhujiang Hospital, Southern Medical University, Guangdong, China.

\section{References}

[1] SIEGEL R, NAISHADHAM D, JEMAL A. Cancer statistics, 2013 CA: a cancer journal for clinicians 2013; 63, 11-30.

[2] BAST RC, JR., HENNESSY B, MILLS GB. The biology of ovarian cancer: new opportunities for translation Nature reviews Cancer 2009; 9, 415-428. http://dx.doi.org/10.1038/ $\underline{\operatorname{nrc} 2644}$

[3] HU X, MACDONALD DM, HUETTNER PC, FENG Z, EL NAQA IM et al. A miR-200 microRNA cluster as prognostic marker in advanced ovarian cancer Gynecologic oncology 2009; 114, 457-464. http://dx.doi.org/10.1016/j. ygyno.2009.05.022

[4] EITAN R, KUSHNIR M, LITHWICK-YANAI G, DAVID $\mathrm{MB}$, HOSHEN $\mathrm{M}$ et al. Tumor microRNA expression patterns associated with resistance to platinum based chemotherapy and survival in ovarian cancer patients Gynecologic oncology 2009;114, 253-259. http://dx.doi.org/10.1016/j. ygyno.2009.04.024
[5] GUO R, WU Q, LIU F, WANG Y. Description of the CD133+ subpopulation of the human ovarian cancer cell line OVCAR3 Oncology reports 2011; 25, 141-146.

[6] WU Q, GUO R, LIN M, ZHOU B, WANG Y. MicroRNA-200a inhibits CD133/1+ ovarian cancer stem cells migration and invasion by targeting E-cadherin repressor ZEB2 Gynecologic oncology 2011; 122, 149-154. http://dx.doi.org/10.1016/j. ygyno.2011.03.026

[7] SEVE P, DUMONTET C. Is class III beta-tubulin a predictive factor in patients receiving tubulin-binding agents? The lancet oncology 2008; 9, 168-175. http://dx.doi.org/10.1016/ $\underline{\text { S1470-2045(08)70029-9 }}$

[8] FENG X, WANG Z, FILLMORE R, XI Y. MiR-200, a new star miRNA in human cancer Cancer letters 2014; 344, 166-173. http://dx.doi.org/10.1016/j.canlet.2013.11.004

[9] MATEESCU B, BATISTA L, CARDON M, GRUOSSO T, DE FERAUDY Y et al. miR-141 and miR-200a act on ovarian tumorigenesis by controlling oxidative stress response Nature medicine 2011; 17, 1627-1635. http://dx.doi.org/10.1038/ $\underline{\text { nm. } 2512}$

[10] HAN Y, HUANG H, XIAO Z, ZHANG W, CAO Y et al. Integrated analysis of gene expression profiles associated with response of platinum/paclitaxel-based treatment in epithelial ovarian cancer PloS one 2012; 7, e52745. http://dx.doi. org/10.1371/journal.pone.0052745

[11] CANCER GENOME ATLAS RESEARCH N. Integrated genomic analyses of ovarian carcinoma Nature 2011; 474, 609-615. http://dx.doi.org/10.1038/nature10166

[12] LEE SH, NAM HJ, KANG HJ, KWON HW, LIM YC. Epigallocatechin-3-gallate attenuates head and neck cancer stem cell traits through suppression of Notch pathway European journal of cancer 2013; 49, 3210-3218. http://dx.doi. org/10.1016/j.ejca.2013.06.025

[13] KUMAR SM, LIU S, LU H, ZHANG H, ZHANG PJ et al. Acquired cancer stem cell phenotypes through Oct4-mediated dedifferentiation Oncogene 2012; 31, 4898-4911. http:// dx.doi.org/10.1038/onc.2011.656

[14] VATHIPADIEKAL V, SAXENA D, MOK SC, HAUSCHKA PV, OZBUN L et al. Identification of a potential ovarian cancer stem cell gene expression profile from advanced stage papillary serous ovarian cancer PloS one 2012; 7, e29079. http://dx.doi. org/10.1371/journal.pone.0029079

[15] HU L, MCARTHUR C, JAFFE RB. Ovarian cancer stem-like side-population cells are tumorigenic and chemoresistant British journal of cancer 2010; 102, 1276-1283. http://dx.doi. org/10.1038/sj.bjc.6605626

[16] COCHRANE DR, HOWE EN, SPOELSTRA NS, RICHER JK. Loss of miR-200c: A Marker of Aggressiveness and Chemoresistance in Female Reproductive Cancers Journal of oncology 2010; 2010, 821717.

[17] FENG B, WANG R, SONG HZ, CHEN LB. MicroRNA-200b reverses chemoresistance of docetaxel-resistant human lung adenocarcinoma cells by targeting E2F3 Cancer 2012; 118, 3365-3376.

[18] LI L, BHATIA R. Stem cell quiescence Clinical cancer research : an official journal of the American Association for Cancer Research 2011 17, 4936-4941. 
[19] LIU P, KUMAR IS, BROWN S, KANNAPPAN V, TAWARI PE et al. Disulfiram targets cancer stem-like cells and reverses resistance and cross-resistance in acquired paclitaxel-resistant triple-negative breast cancer cells British journal of cancer 2013; 109, 1876-1885. http://dx.doi.org/10.1038/bjc.2013.534

[20] WANG L, MEZENCEV R, BOWEN NJ, MATYUNINA LV, MCDONALD JF. Isolation and characterization of stemlike cells from a human ovarian cancer cell line Molecular and cellular biochemistry 2012; 363, 257-268. http://dx.doi. org/10.1007/s11010-011-1178-6
[21] SCHIFF PB, FANT J, HORWITZ SB. Promotion of microtubule assembly in vitro by taxol Nature 1979; 277, 665-667. http://dx.doi.org/10.1038/277665a0

[22] KATSUMATA N, YASUDA M, TAKAHASHI F, ISONISHI S, JOBO T et al. Dose-dense paclitaxel once a week in combination with carboplatin every 3 weeks for advanced ovarian cancer: a phase 3, open-label, randomised controlled trial Lancet 2009; 374, 1331-1338. http://dx.doi.org/10.1016/ S0140-6736(09)61157-0 


\title{
Supplementary Information
}

\section{Upregulation of microRNA-200a associates with tumor proliferation, CSCs phenotype and chemosensitivity in ovarian cancer}

\author{
N. LIU ${ }^{1,2}$, L. ZHONG ${ }^{1}$, J. ZENG ${ }^{1}$, X. ZHANG ${ }^{1}$, Q. YANG ${ }^{1}$, D. LIAO ${ }^{1}$, Y. WANG ${ }^{3}$, G.CHEN ${ }^{1}$, Y. WANG ${ }^{1, *}$
}

${ }^{1}$ Department of Obstetrics and Gynecology, Zhujiang Hospital, Southern Medical University, Guangzhou, China; ${ }^{2}$ Department of Gynecology, Shanghai First Maternity and Infant Hospital Affiliated to Tong Ji University, Shanghai, China; ${ }^{3}$ School of Basic Medical Sciences, Cancer Research Institute, Southern Medical University, Guangzhou, China

*Correspondence: yifengw1963@sina.com

The sequence information of plasmid pLV-hsa-miR-200a is as follows: tGTTTTGAGACTATAAATATCCCTTGGAGAAAAGCCTTGTTAACGCGCGGTGACCCTCGA GAACCCGGGGGATCCTTTTTATTTAACGCGAATTAATTCTGTGGAATGTGTGTCAGTTAG GGTGTGGAAAGTCCCCAGGCTCCCCAGCAGGCAGAAGTATGCAAAGCATGCATCTCAATT AGTCAGCAACCAGGTGTGGAAAGTCCCCAGGCTCCCCAGCAGGCAGAAGTATGCAAAGCA TGCATCTCAATTAGTCAGCAACCATAGTCCCGCCCCTAACTCCGCCCATCCCGCCCCTAA CTCCGCCCAGTTCCGCCCATTCTCCGCCCCATGGCTGACTAATTTTTTTTATTTATGCAG AGGCCGAGGCCGCCTCTGCCTCTGAGCTATTCCAGAAGTAGTGAGGAGGCTTTTTTGGAG GCCTAGGCTTTTGCAAAAAGCTCCCGGCTAGCATGACCGAGTACAAGCCCACGGTGCGCC TCGCCACCCGCGACGACGTCCCCAGGGCCGTACGCACCCTCGCCGCCGCGTTCGCCGACT ACCCCGCCACGCGCCACACCGTCGATCCGGACCGCCACATCGAGCGGGTCACCGAGCTGC AAGAACTCTTCCTCACGCGCGTCGGGCTCGACATCGGCAAGGTGTGGGTCGCGGACGACG GCGCCGCGGTGGCGGTCTGGACCACGCCGGAGAGCGTCGAAGCGGGGGCGGTGTTCGCCG AGATCGGCCCGCGCATGGCCGAGTTGAGCGGTTCCCGGCTGGCCGCGCAGCAACAGATGG AAGGCCTCCTGGCGCCGCACCGGCCCAAGGAGCCCGCGTGGTTCCTGGCCACCGTCGGCG TCTCGCCCGACACCAGGCaAGGGTCTGGGCAGCGCGTCGTGCTCCCGGAGTGAGCGCCGA GCGCGCGGGTGCCGCTTCTGAGACTCGCGCC

The sequence information of plasmid pLV-miR-control is as follows: tGTTTTGAGACTATAAATATCCCTTGGAGAAAAGCCTTGTTAACGCGCGGTGACCCTCGA GAACCCGGGGGATCCTTTTTATTTAACGCGAATTAATTCTGTGGAATGTGTGTCAGTTAG GGTGTGGAAAGTCCCCAGGCTCCCCAGCAGGCAGAAGTATGCAAAGCATGCATCTCAATT AGTCAGCAACCAGGTGTGGAAAGTCCCCAGGCTCCCCAGCAGGCAGAAGTATGCAAAGCA TGCATCTCAATTAGTCAGCAACCATAGTCCCGCCCCTAACTCCGCCCATCCCGCCCCTAA CTCCGCCCAGTTCCGCCCATTCTCCGCCCCATGGCTGACTAATTTTTTTTATTTATGCAG AGGCCGAGGCCGCCTCTGCCTCTGAGCTATTCCAGAAGTAGTGAGGAGGCTTTTTTGGAG GCCTAGGCTTTTGCAAAAAGCTCCCGGCTAGCATGACCGAGTACAAGCCCACGGTGCGCC TCGCCACCCGCGACGACGTCCCCAGGGCCGTACGCACCCTCGCCGCCGCGTTCGCCGACT ACCCCGCCACGCGCCACACCGTCGATCCGGACCGCCACATCGAGCGGGTCACCGAGCTGC AAGAACTCTTCCTCACGCGCGTCGGGCTCGACATCGGCAAGGTGTGGGTCGCGGACGACG GCGCCGCGGTGGCGGTCTGGACCACGCCGGAGAGCGTCGAAGCGGGGGCGGTGTTCGCCG AGATCGGCCCGCGCATGGCCGAGTTGAGCGGTTCCCGGCTGGCCGCGCAGCAACAGATGG AAGGCCTCCTGGCGCCGCACCGGCCCAAGGAGCCCGCGTGGTTCCTGGCCACCGTCGGCG TCTCGCCCGACACCAGGCAAGGGTCTGGGCAGCGCGTCGTGCTCCCGGAGTGAGCGCCGA GCGCGCGGGTGCCGCTTCTGAGACTCGCGCC 


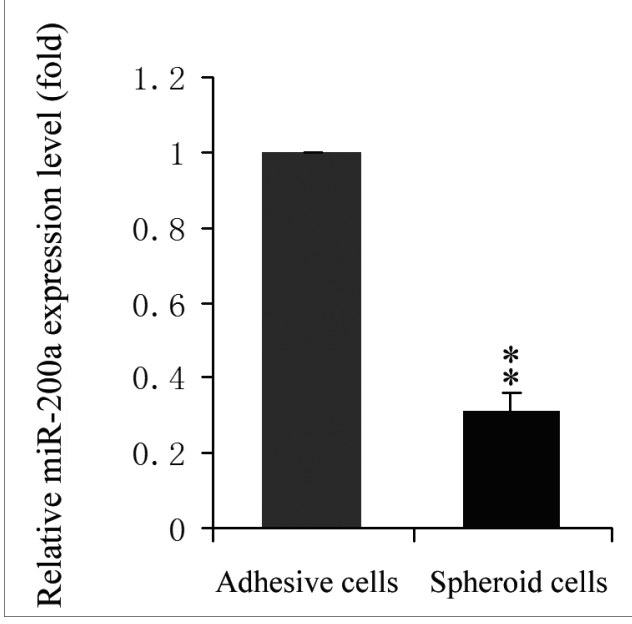

Figure S2 